\title{
Erratum to: Effect of Surface Area on Corrosion Properties of Magnesium for Biomaterials
}

\author{
Woo-Cheol Kim, Kwon-Hoon Han, Jung-Gu Kim, Seok-Jo Yang, Hyun-Kwang Seok, Hyung-Seop Han, \\ and Young-Yul Kim
}

Erratum to : Met. Mater. Int., Vol. 19, No. 5, pp.1131-1137

doi: 10.1007/s12540-013-5032-0

The original version of the article was published in Met. Mater. Int. 19(5) (2013), pp. 1131-1137. Unfortunately, the original version of this article contains a mistake in the affiliation section. The affiliation for Woo-Cheol Kim, Kwon-Hoon Han, Jung-Gu Kim is Sungkyunkwan University, Department of Advanced Materials Engineering, 300 Chunchun-Dong, Jangan-Gu, Suwon 440-746, Korea. 